PHYSICAL REVIEW E 93, 049902(E) (2016)

\title{
Publisher's Note: Explosive percolation transitions in growing networks
}

[Phys. Rev. E 93, 032316 (2016)]

S. M. Oh, S.-W. Son, and B. Kahng

(Received 6 April 2016; published 15 April 2016)

DOI: 10.1103/PhysRevE.93.049902

This paper was published online on 17 March 2016 with an incorrect figure. Figure 1 has been replaced as of 22 March 2016. The figure is correct in the printed version of the journal. 